\title{
Integrative analysis of metabolomic and transcriptomic data reveals metabolic alterations in glioma patients
}

Yingying Shi ${ }^{1,2,3}$, Daling Ding ${ }^{4}$, Liwei Liu ${ }^{1,2,3}$, Zhuolun $\mathrm{Li}^{1,2,3}$, Lihua Zuo ${ }^{1,2,3}$, Lin Zhou $^{1,2,3}$, Qiuzheng Du ${ }^{1,2,3}$, Ziwei Jing ${ }^{1,2,3}$, Xiaojian Zhang ${ }^{1,2,3 *}$, Zhi Sun ${ }^{1,2,3 *}$

1 Department of Pharmacy, the First Affiliated Hospital of Zhengzhou University, Zhengzhou, Henan Province, 450052, P. R. China.

2 Henan Key Laboratory of Precision Clinical Pharmacy, Zhengzhou, Henan Province, 450052, P. R. China.

3 Henan Engineering Research Center of Clinical Mass Spectrometry for Precision Medicine, Zhengzhou, Henan Province, 450052, P. R. China

4 Department of Neurosurgery, The First Affiliated Hospital of Zhengzhou University, Zhengzhou, Henan Province, 450052, P. R. China

\section{Corresponding Authors:}

* Zhi Sun, Department of Pharmacy, the First Affiliated Hospital of Zhengzhou University, Zhengzhou, 450052, P. R. China. E-mail: sunzhi2013@163.com Xiaojian Zhang, Department of Pharmacy, the First Affiliated Hospital of Zhengzhou University, Zhengzhou, 450052, P.R. China. E-mail: zhangxiaojian_yxb@163.com 


\begin{tabular}{|c|c|}
\hline Contents & Pages \\
\hline $\begin{array}{l}\text { Figure S1 The quality control of the LC-MS analyses in negative-ion } \\
\text { mode }\end{array}$ & $S-3$ \\
\hline $\begin{array}{l}\text { Figure S2 Pattern recognition analysis of metabolomics data in negative- } \\
\text { ion mode }\end{array}$ & S-4 \\
\hline $\begin{array}{l}\text { Figure S3 Correlation network analysis of metabolites identified in } \\
\text { untargeted metabolomics. }\end{array}$ & S-5 \\
\hline $\begin{array}{l}\text { Figure S4 Spearman correlation analysis of overlapping metabolites in } \\
\text { both plasma and urine }\end{array}$ & S-6 \\
\hline Figure S5 Metabolites set enrichment analysis & S-7 \\
\hline $\begin{array}{l}\text { Figure S6 ROC curves of other metabolites except to the same } \\
\text { metabolites in plasma and urine metabolomics }\end{array}$ & S-8 \\
\hline Figure S7 The connected network of metabolites and genes & S-9 \\
\hline $\begin{array}{l}\text { Figure S8 The protein-protein interaction network of the } 1437 \\
\text { differential expression genes in human glioma versus healthy controls }\end{array}$ & S-10 \\
\hline Table S1. Demographic information of the subjects in study & S-11-S12 \\
\hline $\begin{array}{l}\text { Table S2 Significant differential metabolites in plasma between glioma } \\
\text { patients and control groups }\end{array}$ & S-13-S-14 \\
\hline $\begin{array}{l}\text { Table S3 Significant differential metabolites in urine between glioma } \\
\text { patients and control groups }\end{array}$ & S-15-S-17 \\
\hline $\begin{array}{l}\text { Table S4 The key targets identified by integration analysis of } \\
\text { metabolomics and transcriptomics data }\end{array}$ & S-18 \\
\hline Table S5 The KEGG enrichment pathways & S-19 \\
\hline
\end{tabular}



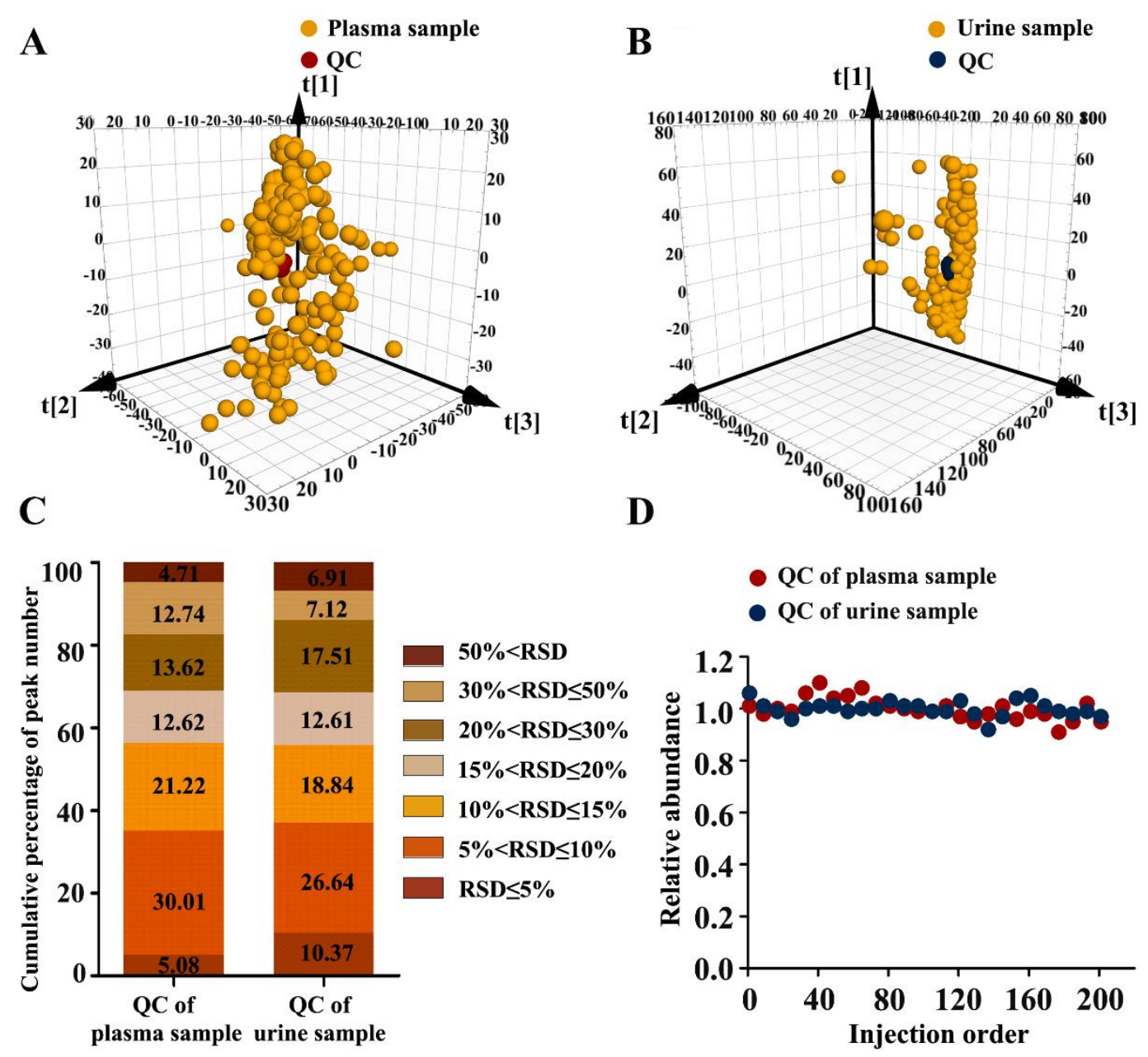

Figure S1. The quality control of the LC-MS analyses in negative-ion mode. Principal component analysis showing the overall variance among analyzed (A) plasma samples and (B) urine samples in negative-ion mode. (C) The distribution of RSD for the compounds detected in the QC samples. (D) The relative abundances with respect to injection order for the internal standards in negative-ion mode. RSD, relative standard deviation; QC, quality control. 

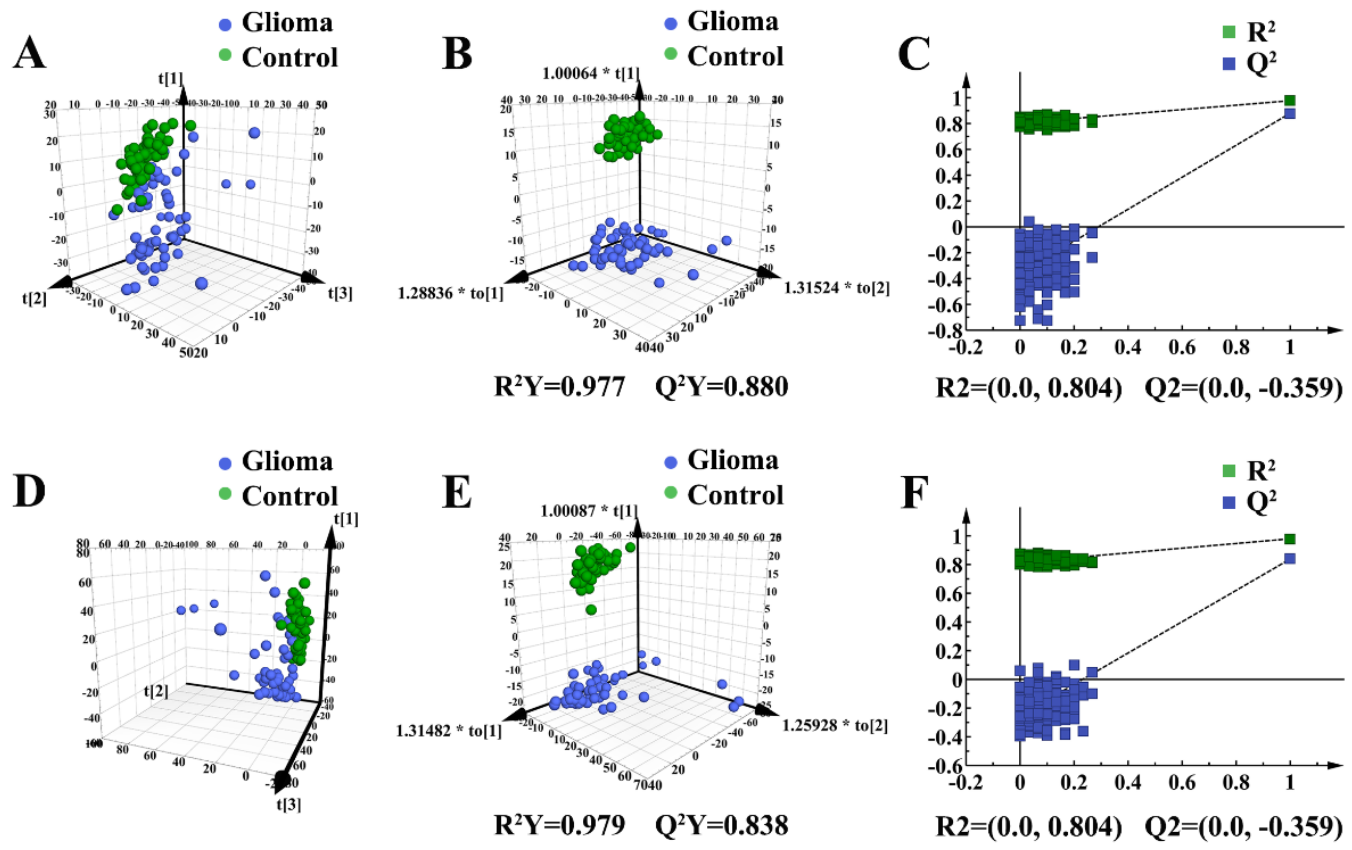

Figure S2. Pattern recognition analys is of metabolomics data in negative-ion mode. Score plots for the PCA model distinguishing gliomas from healthy controls in (A) plasma and (D) urine samples. The OPLSDA score plots for the (B) plasma samples and (E) urine samples in negative-ion mode. The permutation test results for the OPLSDA model among the (C) plasma and (F) urine samples showing that the model was not over-fitting in negative-ion mode. PCA, principal component analysis; OPLSDA, orthogonal partial least-squares discriminant analysis. 


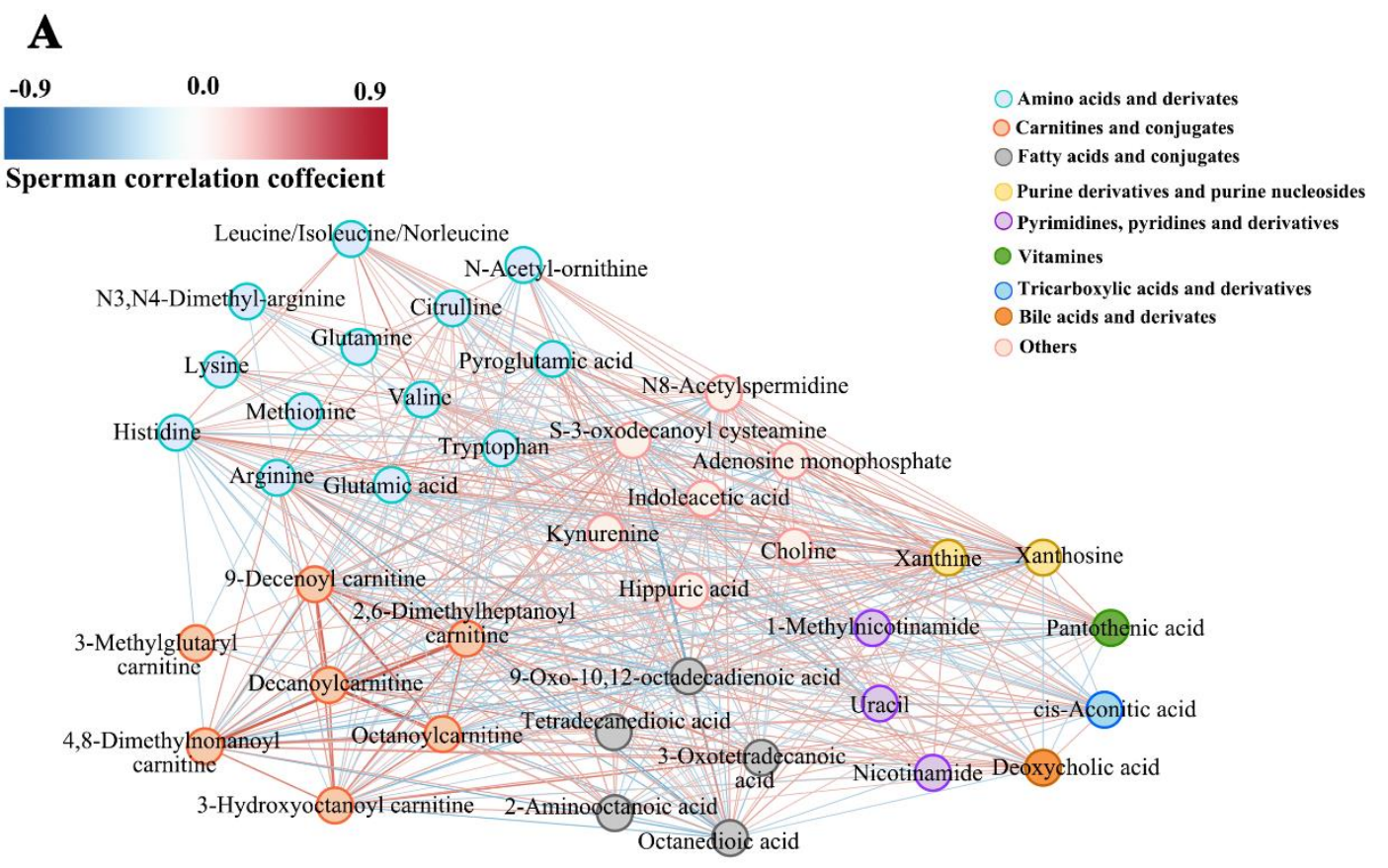

B

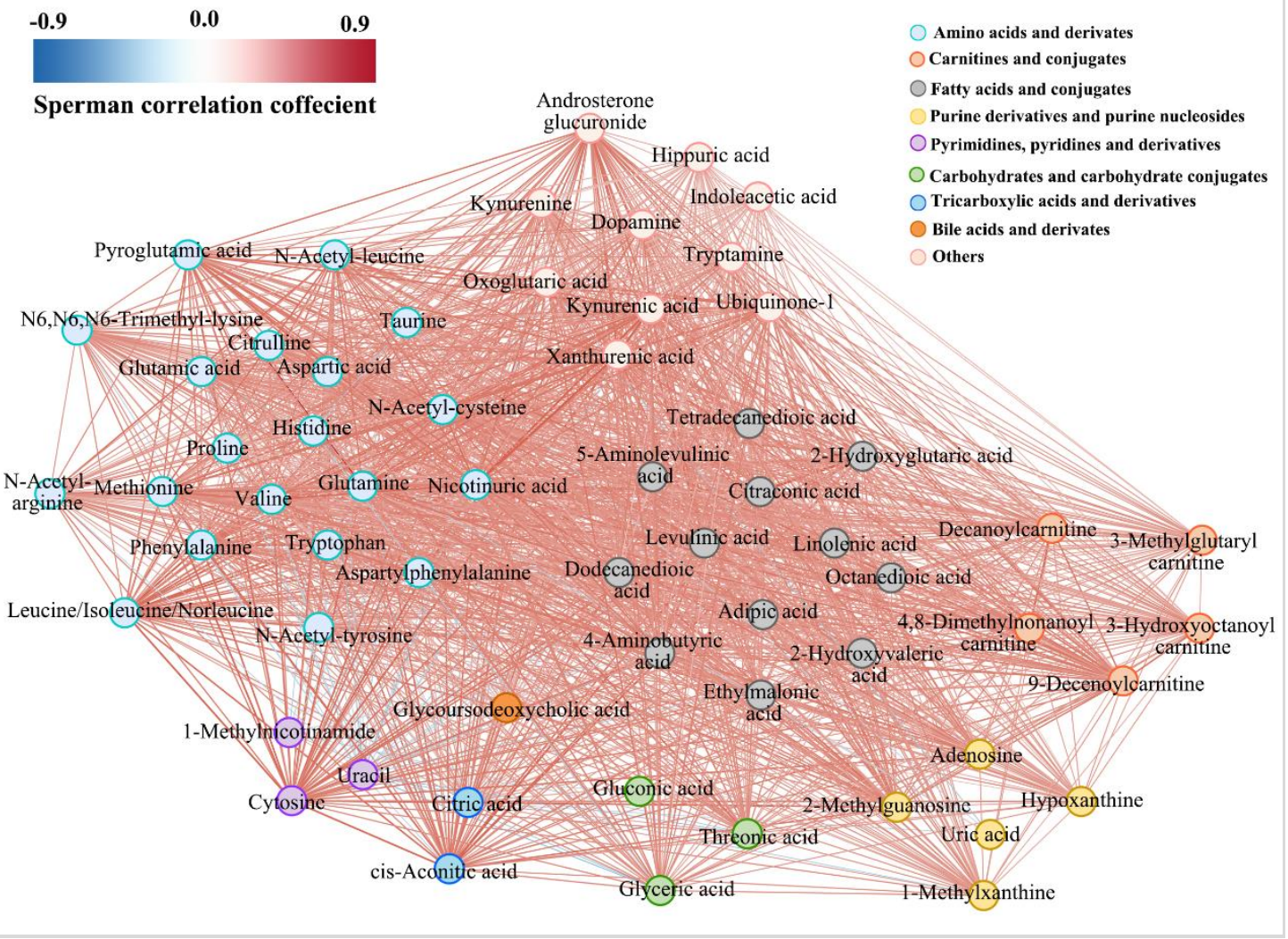

Figure S3. Correlation network analysis of metabolites identified in untargeted metabolomics. (A) Spearman correlation analysis of 40 significantly different metabolites in the plasma samples of the glioma group and the healthy control group. (B) Spearman correlation analysis of 61 significantly different metabolites in glioma patients' and healthy controls' urine samples. 


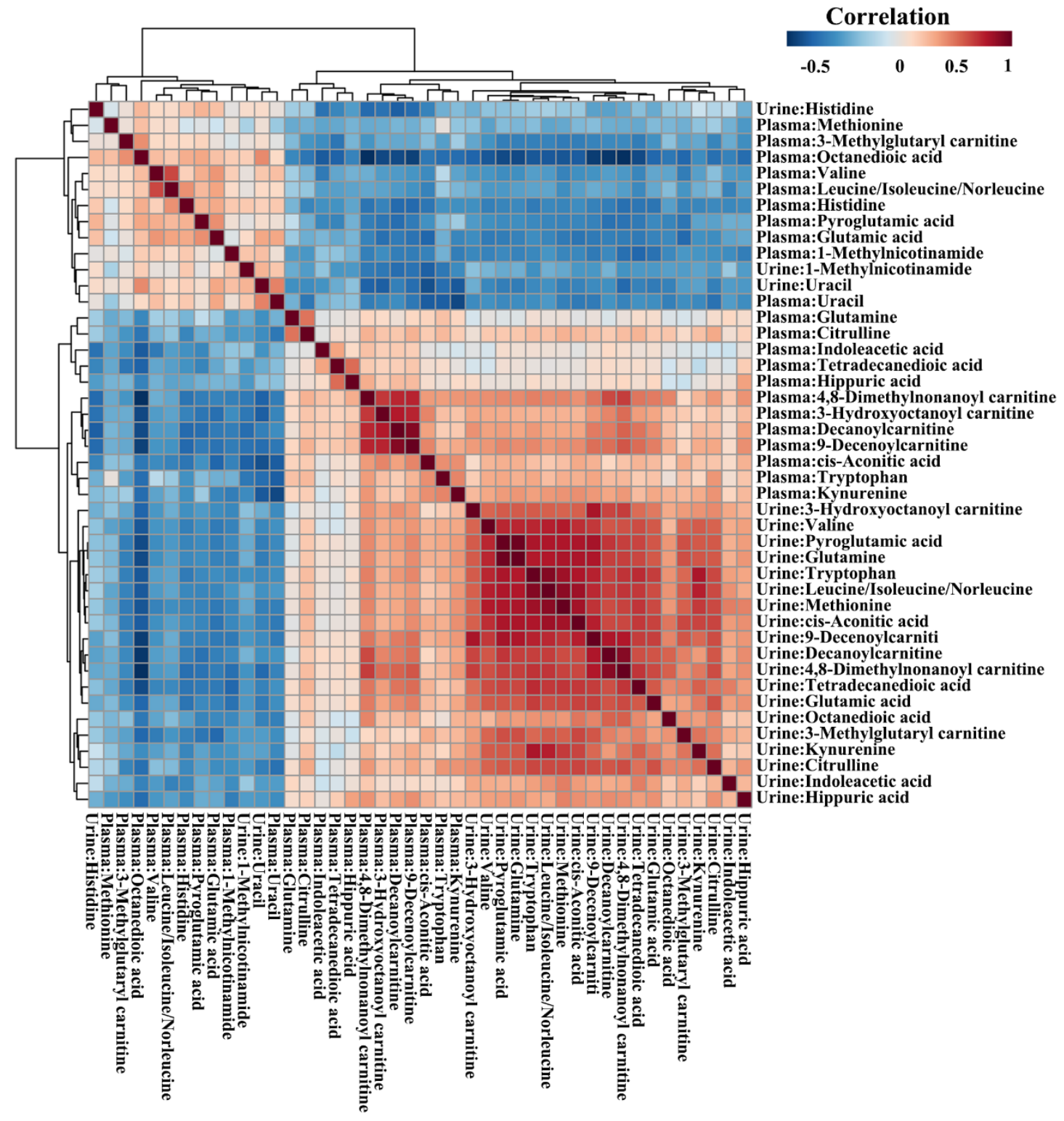

Figure S4. Spearman correlation analysis of overlapping metabolites in both plasma and urine. 

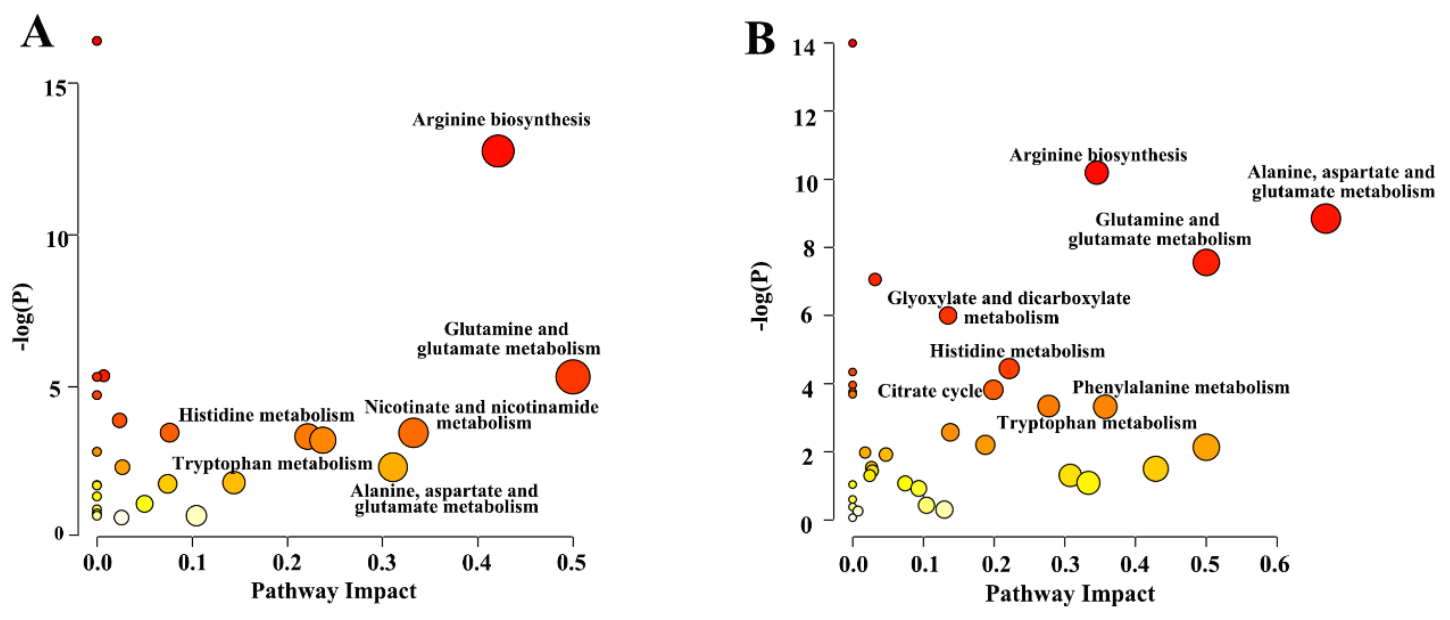

Figure S5. Metabolites set enrichment analysis. (A) Pathway enrichment analysis of plasma metabolites significantly different between the glioma and the healthy controls. (B) Pathway enrichment analysis of urine metabolites significantly different between the glioma patients and the healthy controls. 
$\mathbf{A}$
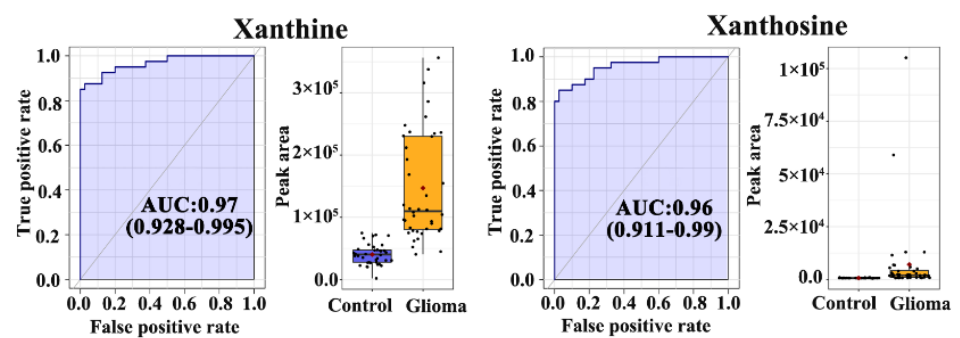

9-Oxo10,12-octadecadienoic acid
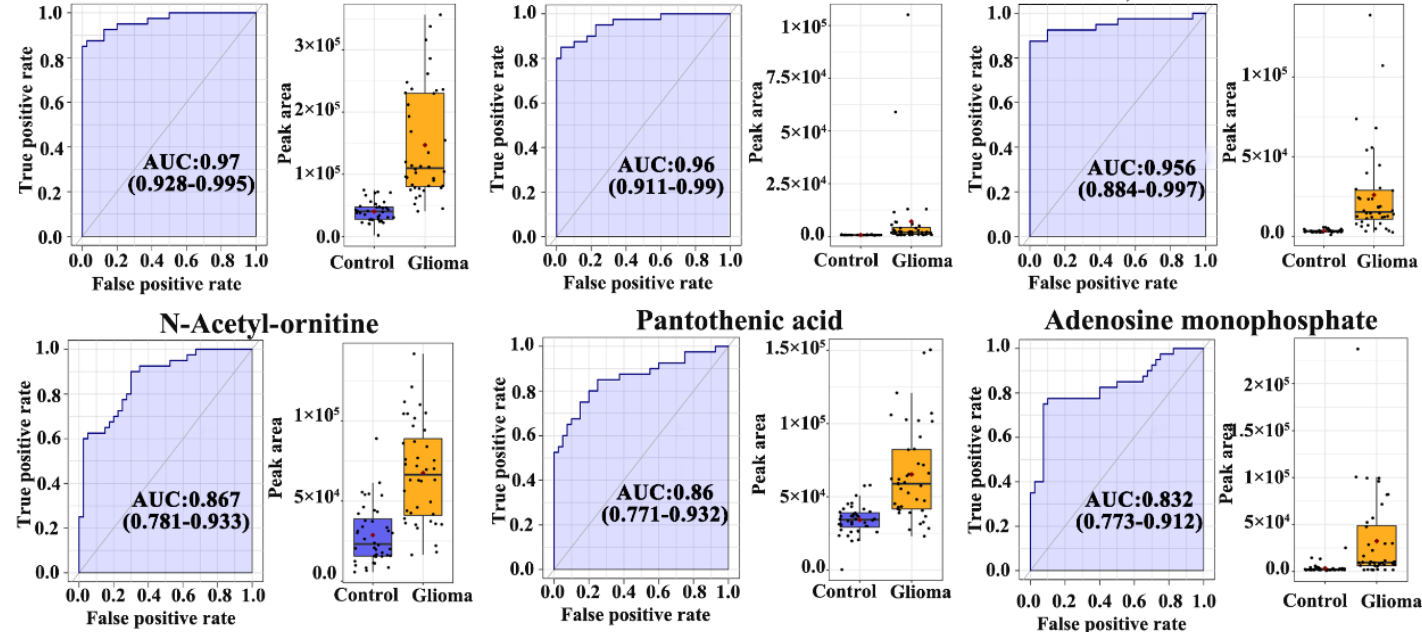

N8-Acetylspermidin
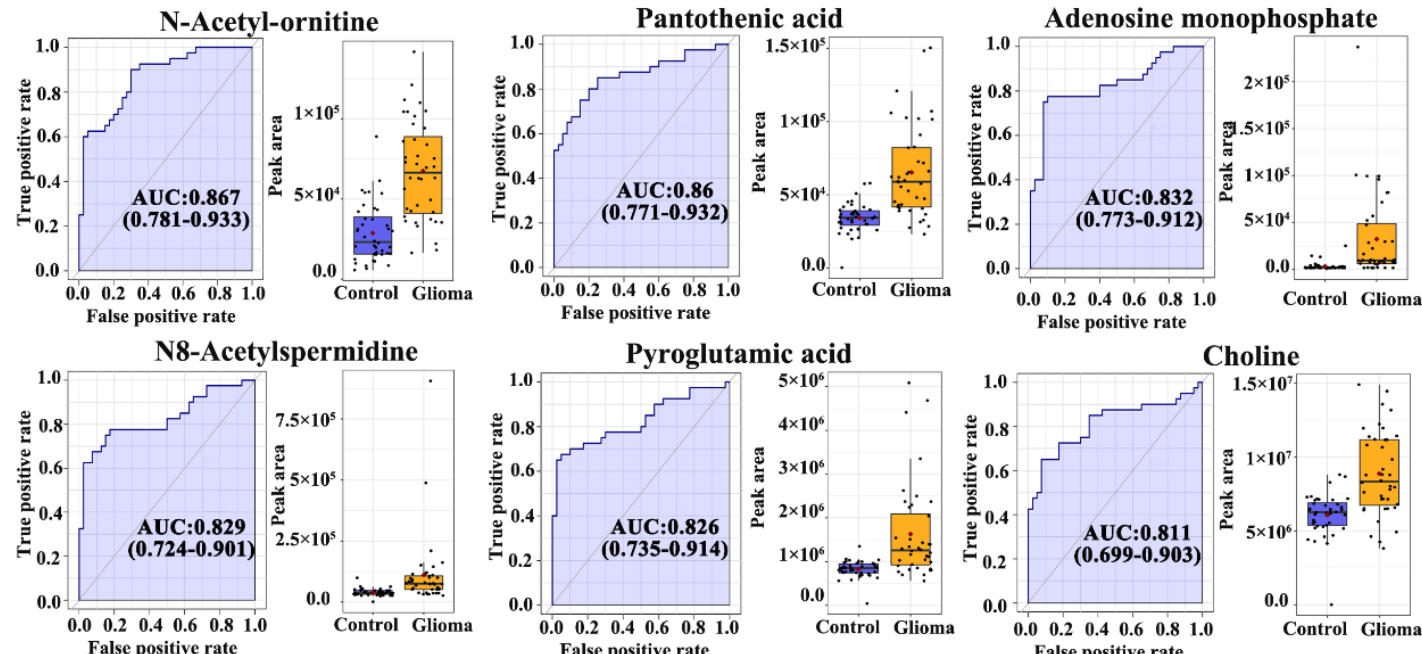

B
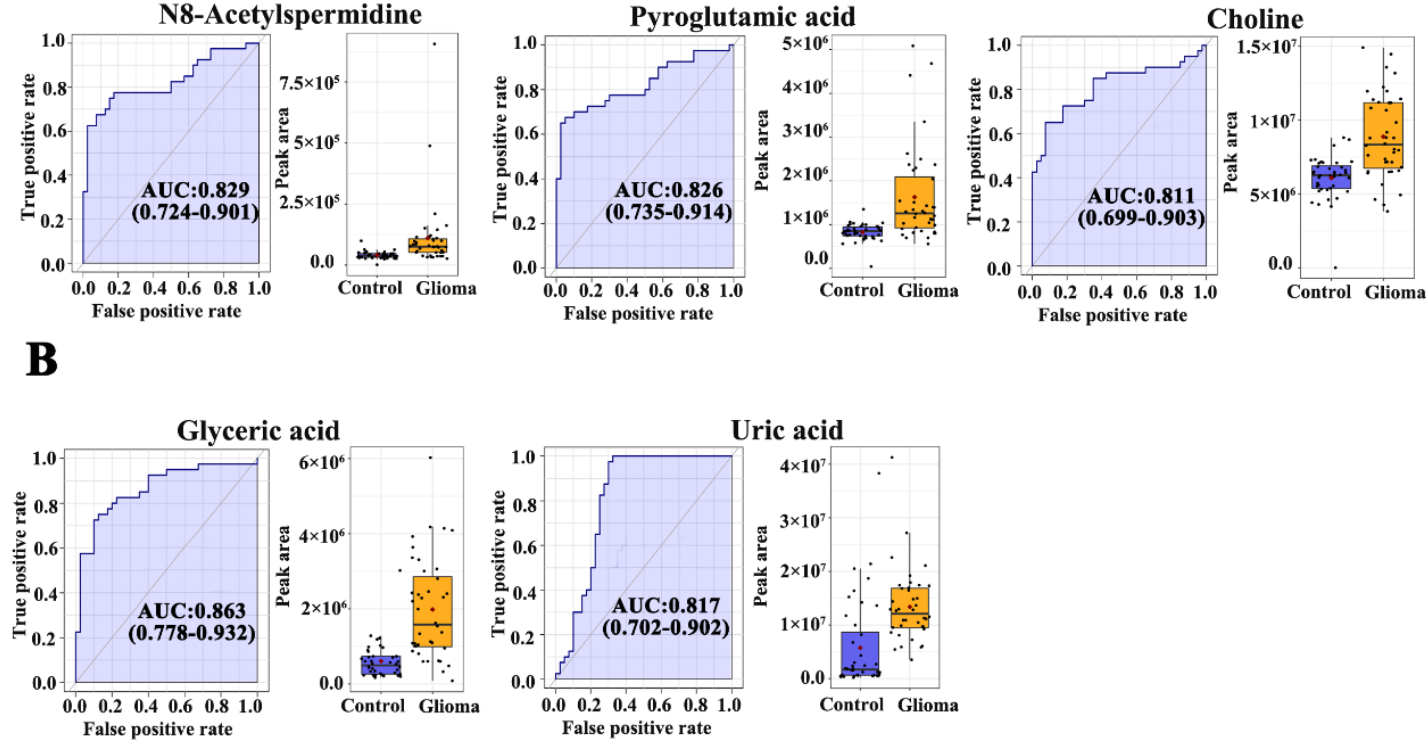

Figure S6. ROC curves of other metabolites except to the same metabolites in plasma and urine metabolomics. (A) ROC curves of plasma metabolites with AUC values exceeding 0.8 in the validation datasets. (B) ROC curves of urine metabolites with AUC values exceeding 0.8 in the validation datasets. 


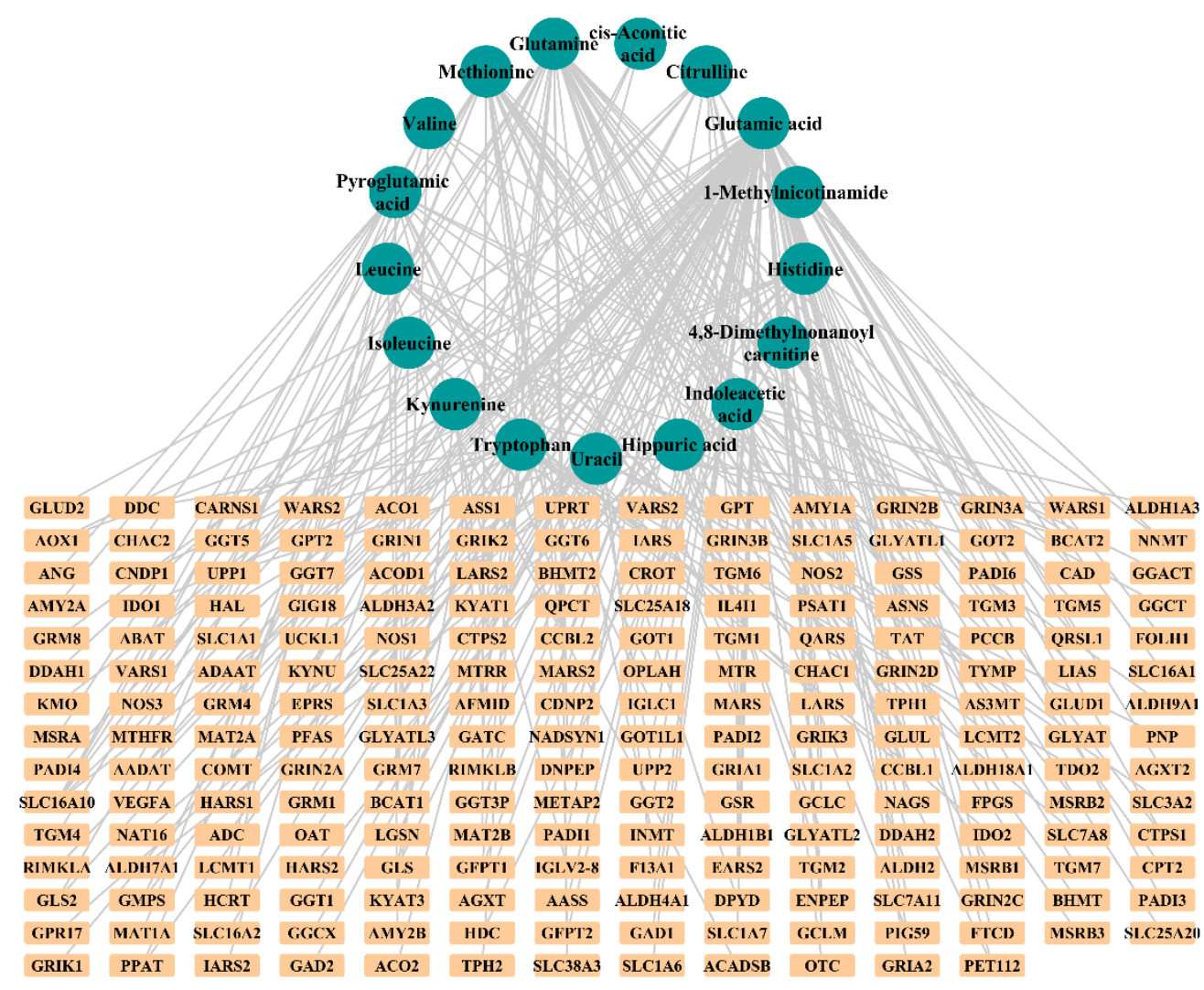

Figure S7. The connected network of metabolites and genes. The green circles node represent the differentially expressed metabolites in the glioma patients versus healthy controls. The orange rectangles represent the genes closely correlated with those metabolites. 


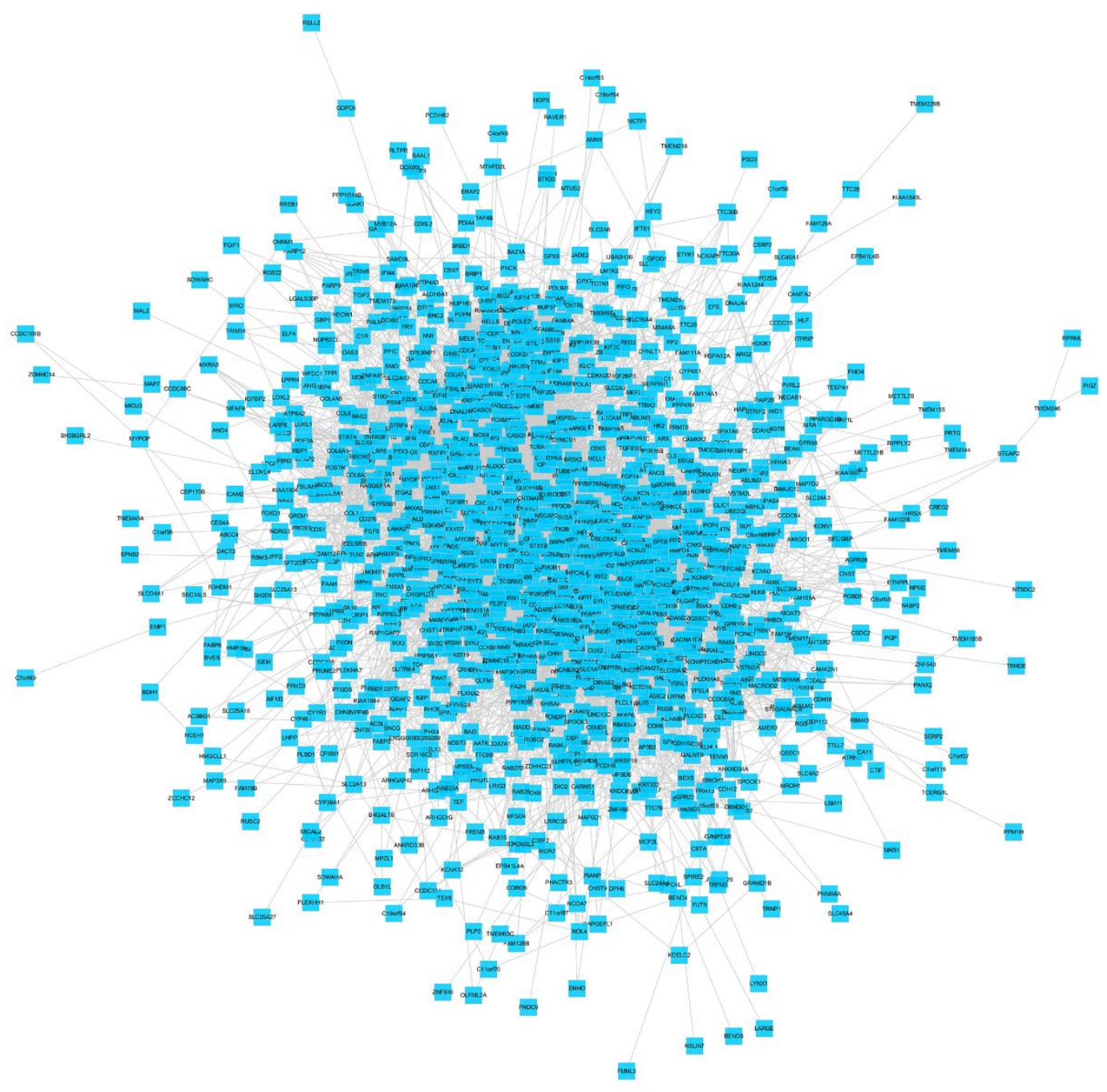

Figure S8. The protein-protein interaction network of the 1437 differential expression genes in human glioma versus healthy controls. 
Table S1. Demographic information of the subjects in study.

\begin{tabular}{|c|c|c|c|c|c|c|}
\hline \multirow{2}{*}{ Category } & \multirow{2}{*}{ Subcategory } & \multicolumn{2}{|c|}{ Discovery cohort } & \multirow{2}{*}{$P^{\mathrm{a}}$} & \multicolumn{2}{|c|}{ Validation cohort } \\
\hline & & $\operatorname{Glioma}(\mathrm{n}=60)$ & Control(n=60) & & $\operatorname{Glioma}(\mathrm{n}=40)$ & Control $(\mathrm{n}=40)$ \\
\hline \multirow[t]{2}{*}{ Gender } & Male & $37(61.7 \%)$ & $33(55.0 \%)$ & 0.463 & $24(60.0 \%)$ & $22(55.0 \%)$ \\
\hline & Female & $23(38.3 \%)$ & $27(45.0 \%)$ & & $16(40.0 \%)$ & $18(45.0 \%)$ \\
\hline Age,years & & $43.5 \pm 17.5$ & $41.5 \pm 12.3$ & 0.463 & $45.7 \pm 20.6$ & $39.0 \pm 12.5$ \\
\hline BMI, $\mathrm{kg} / \mathrm{m}^{2}$ & & $24.4 \pm 4.0$ & $24.2 \pm 2.5$ & 0.704 & $28.3 \pm 2.7$ & $24.6 \pm 4.3$ \\
\hline \multirow[t]{4}{*}{ Pathology } & $\mathrm{PA}$ & $2(3.3 \%)$ & & & $1(2.5 \%)$ & \\
\hline & $\mathrm{DA}, \mathrm{OG}$ & $15(25.0 \%)$ & - & & $11(27.5 \%)$ & - \\
\hline & AA,AEP,AOG & $11(18.3 \%)$ & - & & $13(32.5 \%)$ & - \\
\hline & GBM & $32(53.3 \%)$ & - & & $15(37.5 \%)$ & - \\
\hline \multirow[t]{3}{*}{ IDH1 mutation status } & Negative & $26(43.3 \%)$ & - & & $5(12.5 \%)$ & - \\
\hline & Positive & $7(11.7 \%)$ & - & & $2(5.0 \%)$ & - \\
\hline & Unknown & $27(45 \%)$ & - & & $33(82.5 \%)$ & - \\
\hline \multirow[t]{4}{*}{ WHO grading } & I & $2(3.3 \%)$ & - & & $1(2.5 \%)$ & - \\
\hline & II & $14(23.3 \%)$ & - & & $10(25.0 \%)$ & - \\
\hline & III & $12(20.0 \%)$ & - & & $14(35.0 \%)$ & - \\
\hline & IV & $32(53.3 \%)$ & - & & $15(37.5 \%)$ & - \\
\hline Tumor size(cm3) & & $38.7 \pm 54.5$ & - & & $31.0 \pm 37.2$ & - \\
\hline \multirow[t]{2}{*}{ Recurrence/Progression } & Yes & $7(11.7 \%)$ & - & & $5(12.5 \%)$ & - \\
\hline & No & $53(88.3 \%)$ & - & & $35(87.5 \%)$ & - \\
\hline \multirow[t]{3}{*}{ Smoking status } & Ever a smoker & $3(5.0 \%)$ & $2(3.3 \%)$ & 0.736 & $2(5.0 \%)$ & $1(2.5 \%)$ \\
\hline & Smoker & $11(18.3 \%)$ & $13(21.7 \%)$ & & $6(15.0 \%)$ & $3(7.5 \%)$ \\
\hline & Never & $46(76.7 \%)$ & $45(75.0 \%)$ & & $32(80.0 \%)$ & $36(90.0 \%)$ \\
\hline \multirow[t]{2}{*}{ Steroid use } & Yes & $2(3.33 \%)$ & 0 & 0.159 & $2(5.0 \%)$ & 0 \\
\hline & No & $58(96.7 \%)$ & $60(100 \%)$ & & $38(95.0 \%)$ & $40(100 \%)$ \\
\hline \multirow[t]{2}{*}{ Seizure medication use } & Yes & $8(13.3 \%)$ & 0 & $0.004 * *$ & $8(20.0 \%)$ & 0 \\
\hline & No & $52(86.7 \%)$ & $60(100 \%)$ & & $32(80.0 \%)$ & $40(100 \%)$ \\
\hline \multirow[t]{2}{*}{ Diabetes medication use } & Yes & $6(10 \%)$ & 0 & $0.013^{*}$ & $1(2.5 \%)$ & 0 \\
\hline & No & $54(90 \%)$ & $60(100 \%)$ & & $39(97.5 \%)$ & $40(100 \%)$ \\
\hline Total protein $(\mathrm{g} / \mathrm{L})$ & & $62.5 \pm 7.3$ & $74.7 \pm 4.3$ & $<0.01 * *$ & $62.5 \pm 7.2$ & $72.3 \pm 12.0$ \\
\hline Glucose & & $5.3 \pm 1.9$ & $4.8 \pm 1.0$ & 0.068 & $5.2 \pm 1.4$ & $4.9 \pm 0.5$ \\
\hline Creatine $(\mathrm{mmol} / \mathrm{L})$ & & $61.7 \pm 15.5$ & $67.1 \pm 18.7$ & 0.093 & $64.0 \pm 19.8$ & $64.8 \pm 21.7$ \\
\hline ALT & & $16.8 \pm 10.3$ & $16.9 \pm 7.8$ & 0.931 & $17.6 \pm 11.8$ & $19.6 \pm 12.3$ \\
\hline AST & & $17.9 \pm 5.9$ & $19.2 \pm 4.5$ & 0.166 & $20.7 \pm 11.4$ & $19.9 \pm 6.4$ \\
\hline $\mathrm{TC}(\mathrm{mmol} / \mathrm{L})$ & & $4.1 \pm 0.9$ & $4.5 \pm 0.8$ & $0.027^{*}$ & $4.4 \pm 1.3$ & $4.2 \pm 0.5$ \\
\hline TG $(\mathrm{mmol} / \mathrm{L})$ & & $1.3 \pm 0.8$ & $1.9 \pm 5.9$ & 0.432 & $1.2 \pm 0.5$ & $1.2 \pm 0.7$ \\
\hline $\mathrm{HDL}(\mathrm{mmol} / \mathrm{L})$ & & $1.3 \pm 0.3$ & $1.4 \pm 0.3$ & $0.007 * *$ & $1.3 \pm 0.4$ & $4.2 \pm 17.5$ \\
\hline $\mathrm{LDL}(\mathrm{mmol} / \mathrm{L})$ & & $2.5 \pm 0.8$ & $2.8 \pm 0.8$ & $0.035^{*}$ & $2.9 \pm 1.2$ & $2.6 \pm 0.5$ \\
\hline eGFR (mL/min) & & $106.1 \pm 14.5$ & $106.4 \pm 14.5$ & 0.890 & $103.6 \pm 16.4$ & $105.2 \pm 14.5$ \\
\hline
\end{tabular}

Results are expressed as the number $(\%)$ or means $\pm \mathrm{SD}, * P<0.05,{ }^{*} P<0.01$ compared with healthy controls. "-", not applicable. PA, pilocytic astrocytoma; DA, diffuse astrocytoma; OG, oligodendroglioma; AA, anaplastic astrocytoma; AEP, anaplastic ependymoma; AOG, anaplastic 
oligodendroglioma; GBM, glioblastoma; ALT, alanine aminotransferase; AST, aspartate transaminase; TC, total cholesterol; TG, triglyceride; HDL, high density lipoprotein; LDL, low density lipoprotein; eGFR, estimated glomerular filtration rate. 
Table S2 Significant differential metabolites in plasma between glioma patients and control groups

\begin{tabular}{|c|c|c|c|c|c|c|c|c|c|c|c|c|c|}
\hline No. & Metabolites & $\begin{array}{l}\text { Ion } \\
\text { Mode }\end{array}$ & $\begin{array}{c}\text { Theoretical } \\
\mathrm{m} / \mathrm{z}\end{array}$ & $\begin{array}{c}\text { Experimenta } \\
1 \mathrm{~m} / \mathrm{z} \\
\end{array}$ & $\begin{array}{l}\text { Error } \\
(\mathrm{ppm})\end{array}$ & $\begin{array}{c}\text { RT } \\
(\min )\end{array}$ & $\begin{array}{l}\text { FC(Glioma } \\
\text { /Control) }\end{array}$ & $\begin{array}{c}P- \\
\text { value }\end{array}$ & FDR & VIP & $\begin{array}{l}\mathrm{RSD} \text { in } \\
\mathrm{QC}_{\mathrm{S}}(\%)\end{array}$ & Class & Fragment \\
\hline 1 & Histidine* & $\mathrm{P}$ & 156.07675 & 156.07654 & -1.365 & 1.14 & 1.39 & $3.00 \mathrm{E}-06$ & $1.71 \mathrm{E}-05$ & 1.77 & 20.49 & $\mathrm{AA}$ & $\begin{array}{l}110.07159,95.06081,93.04520,83.06097 \\
82.05315,81.04523,68.05025\end{array}$ \\
\hline 2 & Lysine* & $\mathrm{P}$ & 147.11280 & 147.11270 & -0.709 & 1.16 & 1.19 & $2.41 \mathrm{E}-03$ & $2.47 \mathrm{E}-03$ & 1.09 & 3.74 & AA & $\begin{array}{l}130.08636,129.10228,84.08131,68.05822, \\
67.05492,56.05206\end{array}$ \\
\hline 3 & 1-Methylnicotinamide & $\mathrm{P}$ & 137.07093 & 137.07106 & -0.428 & 1.31 & 2.07 & 2.41E-04 & $3.21 \mathrm{E}-04$ & 1.33 & 10.78 & PPD & $110.06042,96.04488,94.06560,78.03440$ \\
\hline 4 & Choline & $\mathrm{P}$ & 104.10699 & 104.10718 & 1.819 & 1.32 & 1.42 & 4.71E-06 & $1.71 \mathrm{E}-05$ & 1.44 & 3.62 & OTH & $61.08496,60.08158,59.07382,58.06593$ \\
\hline 5 & N8-Acetyls permidine & $\mathrm{P}$ & 188.17573 & 188.17548 & -1.376 & 1.32 & 2.05 & $2.26 \mathrm{E}-05$ & $5.31 \mathrm{E}-05$ & 1.44 & 8.78 & OTH & $\begin{array}{l}\text { 171.14925, 114.09175, 112.11255, 100.07620, } \\
84.08140,58.06596\end{array}$ \\
\hline 6 & Arginine & $\mathrm{P}$ & 175.11895 & 175.11882 & -0.755 & 1.34 & 0.72 & 8.10E-07 & $1.08 \mathrm{E}-05$ & 2.27 & 7.46 & $\mathrm{AA}$ & $\begin{array}{l}158.09245,130.09756,116.07086,113.07148, \\
112.08733,72.08143,70.06586\end{array}$ \\
\hline 7 & Glutamic acid* & $\mathrm{P}$ & 148.06043 & 148.06027 & -1.110 & 1.36 & 1.85 & 1.97E-05 & $5.24 \mathrm{E}-05$ & 1.37 & 30.00 & AA & 130.05006, 102.05542, 85.04827, 84.04501 \\
\hline 8 & N3,N4-Dimethyl-arginine* & $\mathrm{P}$ & 203.15025 & 203.15030 & -0.061 & 1.37 & 1.22 & 2.04E-04 & $2.82 \mathrm{E}-04$ & 1.23 & 7.14 & AA & $\begin{array}{l}\text { 172.10764, 158.12872, 133.09706, 116.07082, } \\
88.08739,70.06580\end{array}$ \\
\hline 9 & Citrulline* & $\mathrm{P}$ & 176.10296 & 176.10275 & -1.237 & 1.38 & 0.77 & $3.92 \mathrm{E}-04$ & $5.06 \mathrm{E}-04$ & 1.17 & 9.28 & AA & $\begin{array}{l}159.07629,116.07076,115.08688,14.05526 \\
113.07124,70.06583\end{array}$ \\
\hline 10 & N-Acetyl-ornithine & $\mathrm{P}$ & 175.10771 & 175.10788 & 0.920 & 1.41 & 2.37 & 3.70E-06 & $1.64 \mathrm{E}-05$ & 1.67 & 27.48 & AA & $116.07092,115.08702,70.06586$ \\
\hline 11 & cis-Aconitic acid & $\mathrm{N}$ & 173.00916 & 173.00783 & -7.694 & 1.52 & 0.57 & 1.49E-06 & $1.49 \mathrm{E}-05$ & 1.52 & 29.46 & TCA & $\begin{array}{l}\text { 154.99715, 129.01801, 111.00732, 85.02798, } \\
83.01247\end{array}$ \\
\hline 12 & Glutamine & $\mathrm{P}$ & 147.07641 & 147.07622 & -1.351 & 1.57 & 0.74 & $1.26 \mathrm{E}-03$ & $1.37 \mathrm{E}-03$ & 1.00 & 17.66 & AA & $130.05005,102.05544,85.04821,84.04499,56.05030$ \\
\hline 13 & Adenosine monophosphate & $\mathrm{P}$ & 348.07036 & 348.06934 & -2.933 & 2.04 & 10.87 & $9.77 \mathrm{E}-04$ & $1.12 \mathrm{E}-03$ & 1.28 & 13.08 & OTH & $136.06195,97.02889$ \\
\hline 14 & Methionine* & $\mathrm{P}$ & 150.05832 & 150.05824 & -0.572 & 2.04 & 1.60 & $5.48 \mathrm{E}-04$ & $6.85 \mathrm{E}-04$ & 1.06 & 5.96 & AA & $\begin{array}{l}\text { 133.03197, 104.05239, 102.05544, 87.02689, } \\
61.01148,56.05034\end{array}$ \\
\hline 15 & Valine & $\mathrm{P}$ & 118.08625 & 118.08632 & 0.549 & 2.09 & 1.23 & 2.20E-05 & $5.51 \mathrm{E}-05$ & 1.43 & 6.24 & AA & $72.08149,58.06590,55.05508$ \\
\hline 16 & Nicotinamide & $\mathrm{P}$ & 123.05528 & 123.05528 & -0.076 & 2.10 & 1.77 & $1.23 \mathrm{E}-04$ & $1.97 \mathrm{E}-04$ & 1.34 & 8.23 & PPD & $106.02911,96.04487,80.05013$ \\
\hline 17 & Pyroglutamic acid* & $\mathrm{P}$ & 130.04986 & 130.04990 & 0.233 & 2.15 & 2.09 & 7.79E-06 & $2.23 \mathrm{E}-05$ & 1.22 & 8.55 & $\mathrm{AA}$ & $85.04829,84.04502,56.05034$ \\
\hline 18 & Xanthine & $\mathrm{P}$ & 153.04070 & 153.04056 & -0.928 & 2.18 & 3.21 & 2.33E-05 & $5.18 \mathrm{E}-05$ & 1.86 & 8.99 & PPN & $110.03526,82.04065,55.02992$ \\
\hline 19 & $\begin{array}{l}\text { Leucine/Isoleucine } \\
\text { /Norleucine }\end{array}$ & $\mathrm{P}$ & 132.10190 & 132.10179 & -0.872 & 2.67 & 1.28 & $1.68 \mathrm{E}-04$ & $2.40 \mathrm{E}-04$ & 1.21 & 19.62 & AA & $86.09702,69.07063$ \\
\hline 20 & Xanthosine & $\mathrm{N}$ & 283.06840 & 283.06787 & -1.898 & 3.17 & 3.62 & $3.81 \mathrm{E}-05$ & $7.62 \mathrm{E}-05$ & 1.45 & 22.80 & PPN & $151.02499,108.01891,61.98698$ \\
\hline 21 & 3-Methylglutaryl carnitine & $\mathrm{P}$ & 290.15981 & 290.15930 & -1.771 & 3.55 & 2.19 & $5.88 \mathrm{E}-04$ & $6.92 \mathrm{E}-04$ & 1.04 & 16.52 & $\mathrm{CC}$ & $\begin{array}{l}231.08664,213.07559,147.06525,129.05498, \\
111.04434,101.06015,85.02901,60.08162\end{array}$ \\
\hline
\end{tabular}




\begin{tabular}{|c|c|c|c|c|c|c|c|c|c|c|c|c|c|}
\hline 22 & Kynurenine & $\mathrm{P}$ & 210.05008 & 210.04980 & -1.348 & 3.58 & 0.66 & $3.82 \mathrm{E}-05$ & 7.27E-05 & 1.54 & 22.32 & OTH & $\begin{array}{l}192.06558,174.05513,163.08698,146.06013, \\
136.07582,120.04462,94.06562,91.05480, \\
65.03941\end{array}$ \\
\hline 23 & Pantothenic acid* & $\mathrm{N}$ & 218.10339 & 218.10254 & -3.924 & 3.62 & 1.72 & $2.47 \mathrm{E}-05$ & $5.21 \mathrm{E}-05$ & 1.41 & 6.89 & VIT & $\begin{array}{l}\text { 146.08099, 116.07060, 99.04373, 88.03886, } \\
71.04868,59.01231\end{array}$ \\
\hline 24 & Tryptophan* & $\mathrm{P}$ & 205.09715 & 205.09715 & -0.752 & 3.77 & 0.80 & $5.58 \mathrm{E}-04$ & $6.76 \mathrm{E}-04$ & 1.17 & 3.56 & AA & $\begin{array}{l}188.07077,159.09184,146.06012,144.08099 \\
132.08095,118.06545,115.05457,91.05475\end{array}$ \\
\hline 25 & Uracil & $\mathrm{P}$ & 113.03455 & 113.03474 & 1.646 & 3.91 & 4.23 & $1.34 \mathrm{E}-04$ & $2.06 \mathrm{E}-04$ & 1.17 & 13.60 & PPD & $95.02450,68.01387$ \\
\hline 26 & 2-Aminooctanoic acid & $\mathrm{P}$ & 160.13320 & 160.13309 & -0.720 & 4.04 & 0.48 & $4.46 \mathrm{E}-06$ & $1.78 \mathrm{E}-05$ & 1.62 & 6.39 & FAC & $114.12811,97.10188,55.05510$ \\
\hline 27 & Hippuric acid & $\mathrm{P}$ & 180.06551 & 180.06612 & 3.334 & 4.21 & 0.45 & $1.26 \mathrm{E}-03$ & $1.40 \mathrm{E}-03$ & 1.04 & 6.66 & OTH & $105.03394,95.04966,81.03423$ \\
\hline 28 & $\begin{array}{l}\text { 3-Hydroxy octanoyl } \\
\text { carnitine }\end{array}$ & $\mathrm{P}$ & 304.21184 & 304.21143 & -1.379 & 4.33 & 0.39 & $6.47 \mathrm{E}-06$ & $2.16 \mathrm{E}-05$ & 2.10 & 11.91 & $\mathrm{CC}$ & $\begin{array}{l}245.13803,227.12727,145.04985,125.09629 \\
103.03947,97.10180,85.02905,60.08163\end{array}$ \\
\hline 29 & Octanedioic acid* & $\mathrm{N}$ & 173.08193 & 173.08066 & -7.350 & 4.45 & 2.49 & $9.21 \mathrm{E}-08$ & $1.84 \mathrm{E}-06$ & 1.71 & 24.71 & FAC & $155.06969,129.09053,111.08009,93.03306$ \\
\hline 30 & Octanoylcarnitine & $\mathrm{P}$ & 288.21693 & 288.21649 & -1.544 & 5.44 & 0.34 & $1.69 \mathrm{E}-06$ & $1.35 \mathrm{E}-05$ & 1.84 & 10.79 & $\mathrm{CC}$ & $\begin{array}{l}229.14374,144.10188,127.11192,109.10130 \text {, } \\
85.02904,60.08160\end{array}$ \\
\hline 31 & Indoleacetic acid & $\mathrm{P}$ & 176.07060 & 176.07042 & -1.051 & 5.51 & 0.24 & 4.27E-03 & 4.27E-03 & 1.21 & 16.15 & $\mathrm{OTH}$ & $130.06534,131.06850,103.05466$ \\
\hline 32 & $\begin{array}{l}\text { 2,6-Dimethylheptanoyl } \\
\text { carnitine }\end{array}$ & $\mathrm{P}$ & 302.23258 & 302.23218 & -1.340 & 5.84 & 0.17 & $6.46 \mathrm{E}-05$ & $1.12 \mathrm{E}-04$ & 1.74 & 12.61 & $\mathrm{CC}$ & $243.15991,85.02897,60.08154$ \\
\hline 33 & 9-Decenoylcarnitine & $\mathrm{P}$ & 314.23258 & 314.23209 & -1.575 & 6.27 & 0.30 & $6.88 \mathrm{E}-06$ & $2.12 \mathrm{E}-05$ & 2.17 & 6.79 & $\mathrm{CC}$ & 255.15923. 153.12772, 135.11703. 85.02902 \\
\hline 34 & Decanoylcarnitine & $\mathrm{P}$ & 316.24823 & 316.24765 & -1.850 & 6.94 & 0.26 & $2.10 \mathrm{E}-06$ & $1.40 \mathrm{E}-05$ & 2.13 & 4.51 & $\mathrm{CC}$ & $257.17459,155.14301,144.10208,85.02904$ \\
\hline 35 & $\begin{array}{l}\text { S-3-oxodecanoyl } \\
\text { cysteamine }\end{array}$ & $\mathrm{P}$ & 246.15222 & 246.15169 & -2.178 & 7.00 & 0.29 & $5.80 \mathrm{E}-08$ & $2.32 \mathrm{E}-06$ & 2.33 & 29.30 & OTH & $\begin{array}{l}229.12556,203.10706,123.11686,61.01145 \\
58.99582\end{array}$ \\
\hline 36 & $\begin{array}{l}\text { 4,8-Dimethylnonanoyl } \\
\text { carnitine }\end{array}$ & $\mathrm{P}$ & 330.26388 & 330.26303 & -2.589 & 7.42 & 0.10 & 7.35E-05 & $1.23 \mathrm{E}-04$ & 1.67 & 5.84 & $\mathrm{CC}$ & $85.02898,71.08620,60.08167$ \\
\hline 37 & $\begin{array}{l}\text { 9-Oxo-10,12- } \\
\text { octadecadienoic acid }\end{array}$ & $\mathrm{P}$ & 295.22677 & 295.22638 & -1.326 & 7.77 & 6.44 & $1.80 \mathrm{E}-03$ & $1.89 \mathrm{E}-03$ & 1.71 & 21.21 & FAC & $\begin{array}{l}277.21622,151.11220,93.070500,79.05495 \\
67.05486,55.05506\end{array}$ \\
\hline 38 & Tetradecanedioic acid & $\mathrm{N}$ & 257.17583 & 257.17526 & -2.226 & 8.82 & 0.44 & $1.64 \mathrm{E}-04$ & $2.43 \mathrm{E}-04$ & 1.07 & 5.17 & FAC & $239.16449,195.17461,61.98672$ \\
\hline 39 & Deoxycholic acid* & $\mathrm{N}$ & 391.28538 & 391.28470 & -1.745 & 9.54 & 0.37 & 4.53E-05 & $8.23 \mathrm{E}-05$ & 1.39 & 5.66 & BAD & $345.27945,343.26431,327.27057$ \\
\hline 40 & 3-Oxotetradecanoic acid & $\mathrm{N}$ & 241.18091 & 241.18008 & -3.474 & 10.80 & 0.40 & $3.20 \mathrm{E}-06$ & $1.60 \mathrm{E}-05$ & 1.51 & 7.46 & FAC & $181.15807,59.01239$ \\
\hline
\end{tabular}

*metabolites matched with commercial available reference standards; RT, rentention time; FC, fold change; $P$-value, the analysis was adjusted by gender, age, BMI, smoking status, steroid use, seizure medication use, and diabetes medication use; FDR, the value was obtained from the false discovery rate (FDR) correction using Benjamini-Hochberg method; VIP, variable importance in the project obtained from glioma patients vs. healthy controls group in discovery cohort; AA,Amino acids and derivatives; CC, Carnitines and conjugates; FAC, Fatty acids and conjugates; PPN, Purine derivatives and purine nucleosides; PPD, Pyrimidines, pyridines and derivatives; VIT, Vitamines; TCA, Tricarboxylic acids and derivatives; BAD, Bile acids and derivatives; OTH, Others. 
Table S3 Significant differential metabolites in urine between glioma patients and control groups

\begin{tabular}{|c|c|c|c|c|c|c|c|c|c|c|c|c|c|}
\hline No. & Metabolites & $\begin{array}{l}\text { Ion } \\
\text { Mode }\end{array}$ & $\begin{array}{c}\text { Theoretical } \\
\mathrm{m} / \mathrm{z}\end{array}$ & $\begin{array}{l}\text { Experimen } \\
\text { tal } \mathrm{m} / \mathrm{z}\end{array}$ & $\begin{array}{l}\text { Error } \\
(\mathrm{ppm})\end{array}$ & $\begin{array}{c}\mathrm{RT} \\
(\min )\end{array}$ & $\begin{array}{l}\text { FC(Glioma } \\
\text { /Control) }\end{array}$ & $P$-value & FDR & VIP & $\begin{array}{l}\mathrm{RSD} \text { in } \\
\mathrm{QC}_{\mathrm{S}}(\%)\end{array}$ & Class & Fragment \\
\hline 1 & N6,N6,N6-Trimethyl-lysine & $\mathrm{P}$ & 189.15975 & 189.15962 & -0.711 & 0.75 & 0.58 & $5.18 \mathrm{E}-06$ & $1.58 \mathrm{E}-05$ & 1.59 & 15.85 & AA & $130.08624,84.08075,60.08102$ \\
\hline 2 & Histidine* & $\mathrm{N}$ & 154.06219 & 154.06198 & -1.427 & 0.75 & 5.96 & 4.65E-04 & $6.16 \mathrm{E}-04$ & 1.39 & 4.77 & $\mathrm{AA}$ & $137.03552,93.04568,110.07211,81.04574$ \\
\hline 3 & Taurine* & $\mathrm{N}$ & 124.00738 & 124.00714 & -1.992 & 0.77 & 0.09 & $1.14 \mathrm{E}-05$ & $2.58 \mathrm{E}-05$ & 1.86 & 6.48 & AA & $106.98063,80.96519,106.98063$ \\
\hline 4 & Gluconic acid* & $\mathrm{N}$ & 195.05102 & 195.05103 & 0.021 & 0.82 & 0.13 & 5.98E-09 & 3.65E-07 & 2.60 & 18.55 & $\mathrm{CBC}$ & $\begin{array}{l}177.04042,135.02998,151.06186,117.01772, \\
87.00864,75.00869,71.01379,59.01382\end{array}$ \\
\hline 5 & Threonic acid & $\mathrm{N}$ & 135.02989 & 135.02971 & -1.382 & 0.82 & 0.49 & $6.11 \mathrm{E}-07$ & 4.14E-06 & 2.05 & 4.61 & $\mathrm{CBC}$ & $\begin{array}{l}117.01913,89.02420,75.00869,72.99307, \\
59.01391\end{array}$ \\
\hline 6 & Aspartic acid & $\mathrm{N}$ & 132.03023 & 132.03006 & -1.295 & 0.84 & 0.41 & $4.38 \mathrm{E}-07$ & $6.68 \mathrm{E}-06$ & 2.16 & 5.89 & AA & $115.00307,88.04026$ \\
\hline 7 & Glyceric acid & $\mathrm{N}$ & 105.01933 & 105.01910 & -2.221 & 0.84 & 2.98 & 2.93E-05 & $5.59 \mathrm{E}-05$ & 1.85 & 8.06 & $\mathrm{CBC}$ & $75.00871,72.99306,59.01382$ \\
\hline 8 & Citrulline* & $\mathrm{P}$ & 176.10296 & 176.10271 & -1.464 & 0.84 & 0.58 & $1.97 \mathrm{E}-04$ & $2.86 \mathrm{E}-04$ & 1.63 & 15.16 & AA & $\begin{array}{l}\text { 159.07646, 131.08987, 130.09744, 116.07036, } \\
113.07103,71.04919,70.06519\end{array}$ \\
\hline 9 & Glutamic acid* & $\mathrm{N}$ & 146.04588 & 146.04568 & -1.377 & 0.88 & 0.23 & $1.36 \mathrm{E}-06$ & $6.38 \mathrm{E}-06$ & 1.30 & 18.42 & AA & $128.03522,102.05596$ \\
\hline 10 & Oxoglutaric acid* & $\mathrm{N}$ & 145.01424 & 145.01404 & -1.424 & 0.89 & 0.17 & $1.98 \mathrm{E}-08$ & $6.04 \mathrm{E}-07$ & 2.49 & 18.97 & OTH & $101.02430,115.00381$ \\
\hline 11 & N-Acetyl-arginine & $\mathrm{P}$ & 217.12951 & 217.12927 & -1.137 & 0.90 & 0.59 & 9.19E-04 & $1.12 \mathrm{E}-03$ & 1.29 & 15.78 & AA & $\begin{array}{l}\text { 175.11952, 158.08112, 116.07053, 112.07561, } \\
60.05589\end{array}$ \\
\hline 12 & 1-Methylnicotinamide & $\mathrm{P}$ & 137.07093 & 137.07083 & -0.799 & 0.94 & 3.19 & $3.16 \mathrm{E}-03$ & $3.57 \mathrm{E}-03$ & 1.03 & 30.00 & PPD & $137.07092,110.06024,94.06507$ \\
\hline 13 & Uric acid & $\mathrm{N}$ & 167.02106 & 167.02083 & -1.397 & 0.98 & 2.02 & 2.66E-04 & 3.77E-04 & 1.34 & 11.54 & PPN & $142.02562,124.01506,69.00935$ \\
\hline 14 & Hypoxanthine* & $\mathrm{P}$ & 137.04578 & 137.04546 & -2.389 & 1.00 & 0.51 & 3.18E-05 & 5.87E-05 & 1.56 & 15.64 & PPN & $119.03519,110.03483,94.03993,55.02946$ \\
\hline 15 & Citric acid & $\mathrm{N}$ & 191.01972 & 191.01945 & -1.444 & 1.01 & 0.39 & $1.21 \mathrm{E}-07$ & 2.46E-06 & 2.64 & 8.85 & TCA & $\begin{array}{l}\text { 173.00894, 147.02982, 129.01917, 111.00865, } \\
87.00864,85.02941,67.01891,57.03454\end{array}$ \\
\hline 16 & Proline* & $\mathrm{P}$ & 116.07060 & 116.07021 & -3.404 & 1.01 & 0.36 & $1.75 \mathrm{E}-04$ & 2.61E-04 & 1.54 & 26.59 & AA & $99.10390,70.06520$ \\
\hline 17 & Dopamine* & $\mathrm{P}$ & 154.08625 & 154.08585 & -2.629 & 1.02 & 0.58 & $5.80 \mathrm{E}-04$ & 7.53E-04 & 1.33 & 18.76 & OTH & 137.05971, 119.04911, 95.06024, 91.05415 \\
\hline 18 & Methionine* & $\mathrm{P}$ & 150.05832 & 150.05797 & -2.371 & 1.02 & 0.49 & $1.11 \mathrm{E}-05$ & 2.61E-05 & 2.08 & 13.39 & AA & $\begin{array}{l}\text { 133.03174, 104.05277, 102.05487, 61.01077, } \\
56.04979\end{array}$ \\
\hline 19 & Adenosine & $\mathrm{P}$ & 268.10403 & 268.10342 & -2.277 & 1.03 & 0.54 & $9.50 \mathrm{E}-06$ & $2.32 \mathrm{E}-05$ & 1.72 & 22.73 & PPN & $\begin{array}{l}\text { 136.06183, 133.04932, } 119.03519,85.02823 \text {, } \\
73.02854\end{array}$ \\
\hline 20 & 5-Aminolevulinic acid & $\mathrm{N}$ & 130.05096 & 130.05055 & -3.202 & 1.05 & 0.48 & $5.68 \mathrm{E}-07$ & 4.95E-06 & 2.00 & 8.29 & FAC & $\begin{array}{l}\text { 113.02422, } 112.02760,102.02760,85.02940, \\
69.03437,58.02980\end{array}$ \\
\hline 21 & cis-Aconitic acid & $\mathrm{N}$ & 173.00916 & 173.00891 & -1.452 & 1.07 & 0.56 & 7.31E-06 & 2.03E-05 & 1.79 & 5.19 & TCA & $129.01924,111.00864,85.02942$ \\
\hline 22 & 2-Hydroxyglutaric acid* & $\mathrm{N}$ & 147.02989 & 147.02980 & -0.657 & 1.12 & 0.60 & $5.41 \mathrm{E}-05$ & $9.16 \mathrm{E}-05$ & 1.53 & 7.29 & FAC & $85.02941,61.98827,59.01379$ \\
\hline
\end{tabular}




\begin{tabular}{|c|c|c|c|c|c|c|c|c|c|c|c|c|c|}
\hline 23 & Citraconic acid* & $\mathrm{N}$ & 129.01933 & 129.01923 & -0.790 & 1.12 & 0.57 & $4.31 \mathrm{E}-06$ & $1.46 \mathrm{E}-05$ & 1.82 & 7.85 & FAC & $85.02942,59.01388$ \\
\hline 24 & Leucine/Is oleucine/Norleucine & $\mathrm{P}$ & 132.10190 & 132.10207 & -3.597 & 1.26 & 0.49 & 4.00E-06 & 1.44E-05 & 1.87 & 15.90 & $\mathrm{AA}$ & $86.09640,69.06995$ \\
\hline 25 & 4-Aminobutyric acid & $\mathrm{N}$ & 102.05605 & 102.05590 & -1.488 & 1.28 & 0.54 & $3.24 \mathrm{E}-05$ & $5.81 \mathrm{E}-05$ & 1.60 & 7.95 & FAC & $84.04519,73.02933,59.01383$ \\
\hline 26 & 2-Methylguanosine & $\mathrm{P}$ & 298.11459 & 298.11444 & -0.520 & 1.32 & 0.54 & $6.04 \mathrm{E}-05$ & 9.70E-05 & 1.61 & 21.01 & PPN & $166.07236,149.04573$ \\
\hline 27 & 1-Methylxanthine & $\mathrm{P}$ & 167.05635 & 167.05588 & -2.825 & 1.56 & 0.16 & $1.07 \mathrm{E}-02$ & $1.11 \mathrm{E}-02$ & 1.16 & 14.74 & PPN & $110.03487,55.02937$ \\
\hline 28 & Cytosine & $P$ & 112.05053 & 112.05012 & -3.734 & 1.70 & 0.45 & $9.23 \mathrm{E}-07$ & 4.69E-06 & 1.96 & 22.50 & PPD & $95.02394,69.04478$ \\
\hline 29 & Kynurenine & $\mathrm{P}$ & 209.09206 & 209.09187 & -0.951 & 1.77 & 0.52 & $8.30 \mathrm{E}-03$ & 8.73E-03 & 1.10 & 16.56 & OTH & $\begin{array}{l}192.06529,174.05489,163.08649,146.05995, \\
136.07571,120.04432,94.06509,74.02365\end{array}$ \\
\hline 30 & Phenylalanine* & $\mathrm{P}$ & 166.08625 & 166.08575 & -3.042 & 1.83 & 0.45 & 3.94E-06 & $1.50 \mathrm{E}-05$ & 1.90 & 17.00 & AA & $\begin{array}{l}\text { 149.05934, 131.04912, 120.08072, 103.05416, } \\
79.05418,51.02336\end{array}$ \\
\hline 31 & Levulinic acid & $\mathrm{N}$ & 115.04006 & 115.03983 & -2.064 & 1.97 & 0.37 & 5.73E-07 & 4.37E-06 & 2.10 & 6.93 & FAC & $97.02941,71.05018,69.03452,53.17003$ \\
\hline 32 & 3-Methylg lutaryl carnitine & $P$ & 290.15981 & 290.15924 & -1.978 & 2.30 & 0.46 & $8.06 \mathrm{E}-04$ & $1.00 \mathrm{E}-03$ & 1.20 & 25.11 & $\mathrm{CC}$ & $\begin{array}{l}231.08630,213.07475,185.08086,144.10179, \\
129.05463,101.05968,85.02838,60.08102\end{array}$ \\
\hline 33 & Ethylmalonic acid & $\mathrm{N}$ & 131.03498 & 131.03474 & -1.847 & 2.46 & 0.50 & $3.76 \mathrm{E}-04$ & $5.21 \mathrm{E}-04$ & 1.35 & 10.86 & FAC & $87.04504,69.03442$ \\
\hline 34 & 2-Hydroxyvaleric acid & $\mathrm{N}$ & 117.05571 & 117.05553 & -1.602 & 2.51 & 0.55 & 7.08E-04 & $9.00 \mathrm{E}-04$ & 1.31 & 21.40 & FAC & $116.92849,71.05018$ \\
\hline 35 & Valine & $\mathrm{P}$ & 118.08625 & 118.08585 & -3.431 & 2.64 & 0.47 & 2.70E-05 & $5.31 \mathrm{E}-05$ & 1.61 & 18.53 & $\mathrm{AA}$ & $72.08083,58.06542,55.05456$ \\
\hline 36 & Xanthurenic acid* & $\mathrm{P}$ & 206.04478 & 206.04446 & -1.574 & 3.02 & 0.37 & 7.28E-07 & 4.04E-06 & 2.08 & 15.16 & $\mathrm{OTH}$ & $188.03413,160.03934,132.04433$ \\
\hline 37 & Tryptophan* & $\mathrm{P}$ & 205.09715 & 205.09703 & -0.606 & 3.05 & 0.44 & $1.57 \mathrm{E}-06$ & $6.40 \mathrm{E}-06$ & 1.89 & 15.39 & AA & $\begin{array}{l}\text { 188.07051. } 170.05989,159.09160,146.05995 \text {, } \\
144.08073,144.08073,118.06500,117.06973 \text {, } \\
115.05409,103.05423\end{array}$ \\
\hline 38 & N-Acetyl-tyrosine & $\mathrm{P}$ & 224.09173 & 224.09135 & -1.715 & 3.17 & 0.44 & 8.90E-05 & $1.36 \mathrm{E}-04$ & 1.13 & 14.87 & AA & $\begin{array}{l}\text { 206.08061, 182.08119, 178.08606, } 165.05461, \\
147.04410,136.07571,119.04913,107.04898\end{array}$ \\
\hline 39 & Nicotinuric acid & $\mathrm{P}$ & 181.06076 & 181.06076 & -0.048 & 3.17 & 0.43 & 5.25E-07 & 5.33E-06 & 2.05 & 17.01 & AA & $163.05009,135.05521,106.02868,51.2345$ \\
\hline 40 & Aspartylphenylalanine & $\mathrm{N}$ & 279.09864 & 279.09860 & -0.161 & 3.24 & 0.56 & 2.94E-03 & $3.39 \mathrm{E}-03$ & 1.14 & 28.04 & AA & $\begin{array}{l}\text { 261.08807, 217.09814, 164.07167, 91.05469, } \\
\text { 72.0089, 147.04515, 113.03551 }\end{array}$ \\
\hline 41 & Kynurenic acid & $\mathrm{P}$ & 190.04986 & 190.04979 & -0.419 & 3.28 & 0.43 & 5.83E-06 & $1.69 \mathrm{E}-05$ & 1.89 & 15.60 & OTH & $162.05489,144.04405,116.04945$ \\
\hline 42 & Tryptamine & $\mathrm{P}$ & 161.10732 & 161.10693 & -2.425 & 3.39 & 0.53 & $2.56 \mathrm{E}-03$ & $3.00 \mathrm{E}-03$ & 1.21 & 17.52 & OTH & 144.08081, 117.06984, 91.05435 \\
\hline 43 & Adipic acid & $\mathrm{N}$ & 145.05063 & 145.05046 & -1.186 & 3.43 & 0.36 & 2.13E-05 & 4.33E-05 & 1.52 & 7.03 & FAC & $127.03989,101.06070,83.05022,81.03435$ \\
\hline 44 & N-Acetyl-cysteine* & $\mathrm{P}$ & 164.03759 & 164.03717 & -2.563 & 3.47 & 0.39 & 2.42E-03 & $2.89 \mathrm{E}-03$ & 1.25 & 18.17 & AA & $\begin{array}{l}\text { 146.02650, 122.02705, 118.03243, 105.00054, } \\
76.02161,58.99533\end{array}$ \\
\hline 45 & Hippuric acid & $\mathrm{P}$ & 180.06551 & 180.06540 & -0.665 & 3.62 & 0.48 & 1.79E-05 & 3.77E-05 & 1.54 & 15.47 & OTH & $162.05458,134.05992,95.04914,53.03896$ \\
\hline 46 & N-Acetyl-leucine* & $\mathrm{P}$ & 174.11246 & 174.11205 & -2.412 & 3.95 & 0.44 & $3.88 \mathrm{E}-05$ & $6.76 \mathrm{E}-05$ & 1.61 & 14.49 & AA & $\begin{array}{l}\text { 156.10164, 132.10184, 128.10695, 86.09360, } \\
69.06998,60.04462\end{array}$ \\
\hline
\end{tabular}




\begin{tabular}{|c|c|c|c|c|c|c|c|c|c|c|c|c|c|}
\hline 47 & Uracil & $\mathrm{P}$ & 113.03455 & 113.03419 & -3.220 & 3.96 & 21.94 & 3.34E-02 & $3.34 \mathrm{E}-02$ & 1.59 & 16.15 & PPD & $96.00813,95.02396,71.04914,70.02878$ \\
\hline 48 & 3-Hydroxyoctanoyl carnitine & $\mathrm{P}$ & 304.21184 & 304.21130 & -1.806 & 4.33 & 0.32 & 8.74E-05 & $1.37 \mathrm{E}-04$ & 1.67 & 18.49 & $\mathrm{CC}$ & $\begin{array}{l}\text { 245.13791, 227.12741, 144.10194, 143.10657, } \\
125.09598,103.03889,85.02835\end{array}$ \\
\hline 49 & Octanedioic acid* & $\mathrm{N}$ & 173.08193 & 173.08168 & -1.457 & 4.44 & 0.26 & 4.26E-04 & 5.77E-04 & 1.49 & 20.28 & FAC & 129.09204, 111.08141, 83.05017, 59.01384 \\
\hline 50 & Dodecanedioic acid & $\mathrm{N}$ & 229.14453 & 229.14453 & -0.011 & 4.53 & 0.48 & $5.86 \mathrm{E}-05$ & $9.66 \mathrm{E}-05$ & 1.38 & 15.86 & FAC & $211.13420,183.13913,59.01380$ \\
\hline 51 & Glutamine & $\mathrm{P}$ & 147.07641 & 147.07603 & -2.643 & 4.71 & 0.41 & $8.10 \mathrm{E}-06$ & 2.15E-05 & 1.70 & 22.64 & AA & $\begin{array}{l}\text { 131.04912, } 120.08072,103.05416,79.05418, \\
51.02336\end{array}$ \\
\hline 52 & Pyroglutamic acid* & $\mathrm{P}$ & 130.04986 & 130.04959 & -2.150 & 4.71 & 0.40 & $1.53 \mathrm{E}-06$ & $6.65 \mathrm{E}-06$ & 1.81 & 16.11 & AA & $84.04438,56.04979$ \\
\hline 53 & Ubiquinone-1 & $\mathrm{P}$ & 251.12778 & 251.12677 & -4.044 & 4.79 & 0.32 & $6.82 \mathrm{E}-07$ & 4.16E-06 & 2.06 & 22.16 & OTH & $\begin{array}{l}233.11703,193.08588,147.08109,105.06976, \\
91.05413,67.05441,55.01820\end{array}$ \\
\hline 54 & Indoleacetic acid & $\mathrm{P}$ & 176.07060 & 176.07016 & -2.528 & 4.82 & 0.49 & 5.44E-03 & $5.82 \mathrm{E}-03$ & 1.18 & 13.82 & OTH & $117.05773,130.06514,103.05415,77.03902$ \\
\hline 55 & 9-Decenoylcarnitine & $\mathrm{P}$ & 314.23258 & 314.23190 & -2.180 & 5.63 & 0.28 & $8.18 \mathrm{E}-06$ & $2.08 \mathrm{E}-05$ & 1.96 & 20.28 & $\mathrm{CC}$ & $\begin{array}{l}255.15869,171.13750,153.12730,135.11684, \\
85.02835\end{array}$ \\
\hline 56 & Decanoylcarnitine & $\mathrm{P}$ & 316.24823 & 316.24747 & -2.419 & 5.86 & 0.25 & 4.63E-06 & 1.49E-05 & 2.06 & 17.83 & $\mathrm{CC}$ & $\begin{array}{l}\text { 257.17447, 173.15332, 144.10204, 85.02837, } \\
155.10305\end{array}$ \\
\hline 57 & Androsterone glucuronide & $\mathrm{N}$ & 465.24939 & 465.24918 & -0.454 & 6.06 & 0.28 & 5.09E-07 & $6.21 \mathrm{E}-06$ & 2.15 & 9.39 & OTH & $447.24188,347.22290,175.02480,71.01378$ \\
\hline 58 & Glycours odeoxycholic acid* & $\mathrm{N}$ & 448.30684 & 448.30756 & 1.591 & 6.19 & 0.32 & $3.58 \mathrm{E}-03$ & 3.97E-03 & 1.16 & 4.66 & BAD & $404.31769,74.02471$ \\
\hline 59 & 4,8-Dimethylnonanoyl carnitine & $\mathrm{P}$ & 330.26388 & 330.26312 & -2.317 & 6.22 & 0.15 & $1.34 \mathrm{E}-05$ & 2.91E-05 & 2.05 & 17.04 & $\mathrm{CC}$ & $144.10210,85.02834,71.08551,60.08103$ \\
\hline 60 & Linolenic acid & $\mathrm{P}$ & 279.23185 & 279.23096 & -3.211 & 6.43 & 0.39 & 2.38E-02 & 2.42E-02 & 1.05 & 17.81 & FAC & $109.10072,95.08549,81.06992,67.05444$ \\
\hline 61 & Tetradecanedioic acid & $\mathrm{N}$ & 257.17583 & 257.17603 & 0.768 & 6.86 & 0.27 & 3.85E-03 & 4.19E-03 & 1.20 & 9.15 & FAC & 213.18494, 195.17555 \\
\hline
\end{tabular}

*metabolites matched with commercial available reference standards; RT, rentention time; FC, fold change; $P$-value, the analys is was adjusted by gender, age, BMI, smoking status, steroid use, seizure medication use, and diabetes medication use; FDR, the value was obtained from the false discovery rate (FDR) correction using Benjamini-Hochberg method. VIP, variable importance in the project obtained from glioma patients vs. healthy controls group in discovery cohort.AA,Amino acids and derivatives; CC, Carnitines and conjugates;FAC, Fatty acids and conjugates; PPN, Purine derivatives and purine nucleosides; PPD, Pyrimidines, pyridines and derivatives; CBC, Carbohydrates and carbohydrate conjugates; TCA, Tricarboxylic acids and derivatives; B AD, Bile acids and derivatives; OTH, Others. 
Table S4 The key targets identified by integration analysis of metabolomic and transcriptomic data

\begin{tabular}{|c|c|c|c|c|}
\hline No. & Target & Protein name & Uniprot ID & Realted metabolites \\
\hline 1 & CNDP1 & Beta-Ala-His dipeptidase & Q96KN2 & Histidine \\
\hline 2 & CARNS 1 & Carnosine synthase 1 & A5YM72 & Histidine \\
\hline 3 & GOT1 & Aspartate aminotransferase, cytoplasmic & $\mathrm{P} 17174$ & Glutamic acid \\
\hline 4 & GAD2 & Glutamate decarboxylase 2 & Q05329 & Glutamic acid \\
\hline 5 & GAD1 & Glutamate decarboxylase 1 & Q99259 & Glutamic acid \\
\hline 6 & GRIN2A & Glutamate receptor ionotropic, NMDA 2A & Q12879 & Glutamic acid \\
\hline 7 & GRIN2C & Glutamate receptor ionotropic, NMDA $2 \mathrm{C}$ & Q14957 & Glutamic acid \\
\hline 8 & GRIK2 & Glutamate receptor ionotropic, kainate 2 & Q13002 & Glutamic acid \\
\hline 9 & GRM1 & Metabotropic glutamate receptor 1 & Q13255 & Glutamic acid \\
\hline 10 & SLC1A1 & Excitatory amino acid transporter 3 & P43005 & Glutamic acid \\
\hline 11 & SLC25A18 & Mitochondrial glutamate carrier 2 & Q9H1K4 & Glutamic acid \\
\hline 12 & SLC1A2 & Excitatory amino acid transporter 2 & P43004 & Glutamic acid \\
\hline 13 & SLC1A6 & Excitatory amino acid transporter 4 & P48664 & Glutamic acid \\
\hline 14 & GRIA2 & Glutamate receptor 2 & P42262 & Glutamic acid \\
\hline 15 & $\mathrm{DDAH} 2$ & $\begin{array}{l}\mathrm{N}(\mathrm{G}), \mathrm{N}(\mathrm{G}) \text {-dimethylarginine } \\
\text { dimethylaminohydrolase } 2\end{array}$ & O95865 & Citrulline \\
\hline 16 & NOS 1 & Nitric oxide synthase, brain & P29475 & Citrulline \\
\hline 17 & UPP2 & Uridine phosphorylase 2 & O95045 & Uracil \\
\hline
\end{tabular}


Table S5 The KEGG enrichment pathways

\begin{tabular}{cccccc}
\hline KEGG ID & Term & Count & P Value & Pop Hits & FDR \\
\hline hsa04724 & Glutamatergic synapse & 8 & $8.90 \mathrm{E}-10$ & 114 & $3.91 \mathrm{E}-08$ \\
hsa05014 & Amyotrophic lateral sclerosis (ALS) & 5 & $2.34 \mathrm{E}-06$ & 50 & $5.16 \mathrm{E}-05$ \\
hsa00410 & beta-Alanine metabolism & 4 & $2.92 \mathrm{E}-05$ & 31 & $4.28 \mathrm{E}-04$ \\
hsa00330 & Arginine and proline metabolis $m$ & 4 & $1.24 \mathrm{E}-04$ & 50 & $1.37 \mathrm{E}-03$ \\
hsa04720 & Long-term potentiation & 4 & $2.85 \mathrm{E}-04$ & 66 & $2.50 \mathrm{E}-03$ \\
hsa04713 & Circadian entrainment & 4 & $8.32 \mathrm{E}-04$ & 95 & $6.08 \mathrm{E}-03$ \\
hsa04080 & Neuroactive ligand-receptor interaction & 5 & $1.87 \mathrm{E}-03$ & 277 & $1.17 \mathrm{E}-02$ \\
hsa00250 & Alanine, aspartate and glutamate metabolism & 3 & $2.20 \mathrm{E}-03$ & 35 & $1.21 \mathrm{E}-02$ \\
hsa05033 & Nicotine addiction & 3 & $2.87 \mathrm{E}-03$ & 40 & $1.40 \mathrm{E}-02$ \\
hsa05030 & Cocaine addiction & 3 & $4.28 \mathrm{E}-03$ & 49 & $1.87 \mathrm{E}-02$ \\
hsa04020 & Calcium signaling pathway & 4 & $5.10 \mathrm{E}-03$ & 179 & $2.03 \mathrm{E}-02$ \\
hsa04730 & Long-term depression & 3 & $6.36 \mathrm{E}-03$ & 60 & $2.31 \mathrm{E}-02$ \\
hsa05031 & Amphetamine addiction & 3 & $7.66 \mathrm{E}-03$ & 66 & $2.57 \mathrm{E}-02$ \\
\hline
\end{tabular}

